\section{Delayed Cosmetic Improvement following Breast Conservation Therapy}

Sir:

$1 /$ e read with great pleasure the interesting article by Patel et al., ${ }^{1}$ and we congratulate the authors for their study comparing outcomes following oncoplastic reduction mammaplasty at various time intervals in relation to radiotherapy. Partial mastectomy defect reconstruction is important to increase indications for breast conservation therapy and to minimize the potential for a poor aesthetic result.

Clough et al. ${ }^{2}$ classified partial mastectomy defects into three groups, with different surgical treatment: (1) large defects in large breasts, which can be repaired by reshaping the breast with oncoplastic reduction mammaplasty; (2) medium defects in smaller breasts, which require local flaps; and (3) large defects in medium to small breasts, for which distant flaps are necessary. The general trend is to reconstruct these defects before radiotherapy, with the benefits of operating on breast tissue that is not irradiated, scarred, or poorly vascularized. The main concern with immediate reconstruction is the potential for positive margins. Staged-immediate reconstruction is a potential alternative, which gives the benefit of reconstruction before irradiation, with the certainty of negative margins, but at the price of a second procedure.

Remodeling of irradiated breast tissue is technically challenging and often associated with surgical complications. ${ }^{3}$ The glandular flaps are poorly vascularized and much less robust, and subsequent scarring and distortion are common in the long term, with progressive deterioration of cosmesis. Where volume asymmetry is the principal issue, a contralateral reduction is probably the best approach and avoids surgical intervention on an irradiated field, unless the radiation damage is not relevant and the patient wishes to undergo reshaping of the irradiated breast and accepts possible complications (Fig. 1).

Where there is marked distortion in the shape of the treated breast, local glandular flaps should be avoided and distant flaps used to bring fresh blood supply to the irradiated breast. A recently developed tool with which to treat the sequelae of breast-conserving therapy is lipofilling. Fat transfer following breast cancer treatment is an indication that is becoming increasingly popular in oncoplastic surgery to improve the morphologic results after partial and total breast reconstruction. It is a simple technique that usually provides a low complication rate and good cosmetic results, especially after radiotherapy, reducing the damage caused by irradiation. Regardless of the effectiveness of this procedure, there are many clinical questions concerning the safety of lipofilling after breast cancer treatment, especially in breast-conserving protocols. A recent multicenter study ${ }^{4}$ confirmed the belief that lipofilling following breast cancer treatment leads to a very low complication rate and does not affect the radiographic follow-up after breast-conserving surgery. Even if they could not provide definitive proof of the safety of fat transfer in terms of cancer recurrence or distant metastasis, lipofilling can be performed in experienced hands, with a cautious oncologic follow-up protocol.

Ideally, partial reconstruction should be undertaken as an immediate procedure following breast-conservation therapy if optimal cosmetic results are to be achieved and maintained in the long term. If this scenario is not feasible, minimal adjustment should be performed in the irradiated breast, such as lipofilling, or contralateral adjustment on the healthy breast (Fig. 2), unless new well-vascularized flaps are provided. DOI: $10.1097 /$ PRS.0b013e3182419a5f
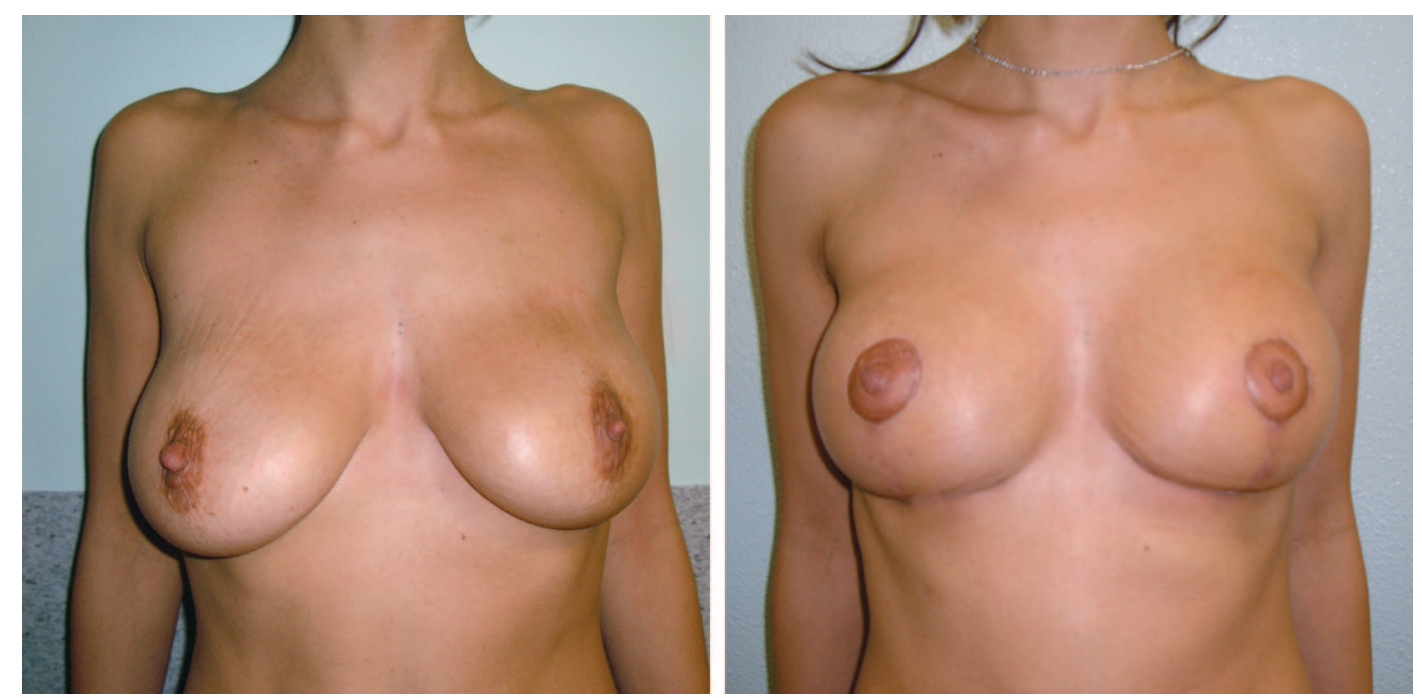

Fig. 1. (Left) Preoperative view of a 34-year-old woman with previous left breast-conserving surgery and radiotherapy. (Right) She successfully underwent delayed oncoplastic reshaping with inferior pedicle mammaplasty. 

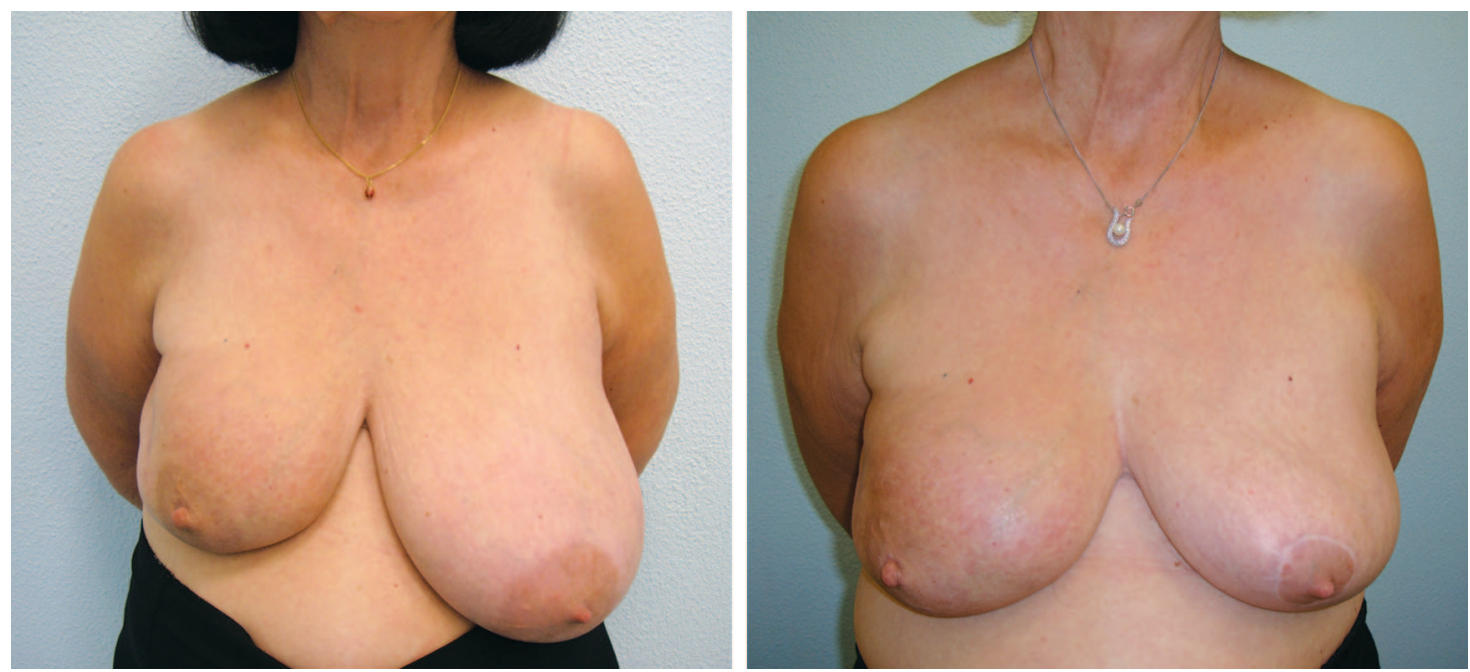

Fig. 2. (Left) Preoperative view of a 62-year-old woman with previous right breast-conserving surgery and radiotherapy. (Right) Appearance 1 year after lipofilling on the right and contralateral reduction mammaplasty.

Stefano Bonomi, M.D. Fernanda Settembrini, M.D. Flavia Sorbi, M.D. Gaetano Musumarra, M.D.

Vincenzo Rapisarda, M.D.

Department of Plastic Reconstructive Surgery and Burn Unit Center

Ospedale Niguarda Ca' Granda Milan, Italy

Correspondence to Dr. Settembrini Department of Plastic Reconstructive Surgery and Burn Unit Center

Ospedale Niguarda Ca' Granda Piazza Ospedale Maggiore, 3 20162 Milano, Italy

fernisettembrini@hotmail.com

\section{DISCLOSURE}

The authors have no financial interest to declare in relation to the content of this communication.

\section{REFERENCES}

1. Patel KM, Hannan CM, Gatti ME, Nahabedian MY. A headto-head comparison of quality of life and aesthetic outcomes following immediate, staged-immediate and delayed oncoplastic reduction mammaplasty. Plast Reconstr Surg. 2011;127: 2167-2175.

2. Clough KB, Kroll SS, Audretsch W. An approach to the repair of partial mastectomy defects. Plast Reconstr Surg. 1999;104: 409-420.

3. Kronowitz SJ, Hunt KK, Kuerer HM, et al. Practical guidelines for repair of partial mastectomy defects using the breast reduction technique in patients undergoing breast conserving therapy. Plast Reconstr Surg. 2007;120:1755-1768.

4. Petit JY, Lohsiriwat V, Clough KB, et al. The oncologic outcome and immediate surgical complications of lipofilling in breast cancer patients: A multicenter study. Milan-Paris-Lyon experience of 646 lipofilling procedures. Plast Reconstr Surg. 2011;128:341-346.

\section{Reply: Delayed Cosmetic Improvement following Breast Conservation Therapy Sir:}

I would like to thank Dr. Settembrini et al. for their comments and observations with regard to our article. We agree that morbidities are increased in the setting of reduction mammaplasty following radiation therapy. Our clinical experience concurs with theirs, and our complication profile is similar. An important fact to remember is that our study protocol was not prospective. Women were not randomized into three treatment arms. As such, we are not advocating for delayed reduction mammaplasty following radiation therapy. The unfortunate reality is that some women with severe mammary hypertrophy have had breast-conservation therapy without being offered the option of immediate or stagedimmediate reduction mammaplasty. It is this group of women that constituted the delayed arm of this study. That said, some notable observations were made.

The conclusions of our study were based on the patient satisfaction data obtained using the BREAST-Q. We found that there were challenges associated with the assessment of satisfaction, especially in the setting of oncoplastic reduction mammaplasty. This is partly because the BREAST-Q contained validated questions for cancer patients and reduction mammaplasty patients; however, there was not a validated scale for cancer patients having reduction mammaplasty. In addition, the dichotomy between patients and surgeons regarding outcomes was evident. Surgeons often judge outcomes based on what they see, whereas patients often judge outcomes based on what they see and how they feel. The ratio between physical and psychological impact following breast surgery is different for different women and is difficult to assess. Oncoplastic reduction mammaplasty clearly has a psychological impact; it is not only visible. There have been several instances in my practice where I felt that a suboptimal outcome was 